\title{
Modélisation d'une machine asynchrone par réseaux de perméances en vue de sa commande
}

\author{
C. Delforge-Delmotte et B. Lemaire-Semail (*) \\ L2EP, École Centrale de Lille, B.P. 49, Cité Scientifique, 59651 Villeneuve d'Ascq Cedex, France
}

(Reçu le 19 janvier 1996, révisé le 24 juin 1996, accepté le 6 septembre 1996)

PACS.07.05.Tp - Computer modeling and simulation

\begin{abstract}
Résumé. - Pour respecter un bon compromis précision-temps de calcul, nous utilisons une méthode de modélisation électromagnétique basée sur une représentation par réseau de perméances. Cette méthode est décrite dans la première partie de l'article : le calcul des éléments du circuit est effectué par la méthode des éléments finis et la saturation prise en compte par l'introduction de perméances variables en fonction du flux. Les couplages électriques et mécaniques sont interprétés grâce à la méthode des bond-graphs dont les grandes lignes sont également rappelées. La méthode est utilisée dans une deuxième partie pour modéliser une machine asynchrone à cage rotorique. La cohérence des résultats obtenus pour la modélisation de la machine seule et les faibles temps de calcul nécessaires, permettent d'envisager l'utilisation de cette méthode pour la simulation d'une machine asynchrone dans son environnement de commande. Les résultats précédents sont alors complétés par l'étude du comportement de la machine commandée vectoriellement.
\end{abstract}

\begin{abstract}
In order to obtain a good compromise accuracy/computation time, we use permeance network method to solve the magnetic equations of an electromagnetic system. This method is explained in the first part of the paper: the network elements are calculated from finite element method, and then, the saturation is considered with flux depending permeances. As for the electric and mechanic coupling, they are represented with the bond-graph method which the main principles are remained. In the second part, the method is applied to a squirrel cage induction machine. The results are discussed and accounting for the low computation time of the method, it is used to study the vector controlled induction machine behaviour.
\end{abstract}

\section{Introduction}

La connaissance du comportement des systèmes électromagnétiques devient, à tous niveaux, une nécessité majeure aussi bien pour leurs concepteurs que pour leurs utilisateurs. À tous niveaux, car le problème ne se pose évidemment pas dans les mêmes termes pour l'ingénieur qui doit prévoir les pertes thermiques dans les têtes de bobines d'un alternateur, ou pour celui qui doit motoriser un bras de robot. Dans un cas, il est nécessaire de prévoir les valeurs locales des grandeurs électromagnétiques, dans l'autre, un comportement global du système incluant la motorisation peut suffire. La méthode de modélisation choisie devra donc être adaptée au cahier des charges de l'exploitation.

$\left({ }^{*}\right)$ Auteur auquel doit être adressée la correspondance 
Parmi les nombreuses méthodes de modélisation électromagnétique, il est possible de dégager deux grandes familles : la famille des méthodes analytiques et celle des "éléments finis" au sens large. Les premières reposent sur des équations où sont mises en jeu des grandeurs globales caractérisant le système telles que, pour une machine électrique, le flux par phase, la tension ou le courant. En opposition, les méthodes de la deuxième famille s'appuient sur des lois et des grandeurs locales telles les champs magnétiques et électriques, ainsi que les caractéristiques internes des matériaux utilisés. Ces spécificités destinent chaque méthode à des applications diversifiées. Dans le cas des machines électriques, les approches analytiques, [1,2], permettent la caractérisation des régimes transitoires et des fonctionnements en environnement électronique complexe. Les méthodes numériques par éléments finis, [3,4], ou différences finies [5], sont plus particulièrement employées dans la caractérisation des machines avec pour finalité la conception assistée par ordinateur.

Compte tenu des temps de calcul importants nécessités par les méthodes numériques, il est rare d'envisager par ce moyen la modélisation de systèmes complexes. Cependant, les hypothèses simplificatrices sur lesquelles reposent les méthodes analytiques classiques (linéarité des matériaux magnétiques, distribution sinusoïdale du champ dans les machines électriques, absence d'effet pelliculaire...) limitent la précision du modèle ; on relève alors sur le système réel des dysfonctionnements non prévus par la simulation. Ceci est d'autant plus manifeste si on utilise le système dans des conditions éloignées des conditions nominales et dans le souci d'obtenir des performances dynamiques de plus en plus grandes.

Afin d'étudier ces fonctionnements, nous avons opté pour une méthode de modélisation basée sur la représentation des systèmes par circuits magnétiques équivalents [6]. Cette méthode peut s'inscrire dans la famille des méthodes numériques, au sens où elle travaille avec des grandeurs "locales" telles les différences de potentiel scalaire magnétique et les flux à travers diverses sections du matériau ; la saturation des matériaux magnétiques pourra donc être prise en compte, tout en limitant la complexité des calculs mis en œuvre. Largement utilisée depuis plusieurs décennies, $[19,20]$, cette méthode trouve un regain d'intérêt dans la modélisation de systèmes complexes de géométrie tridimensionnelle ou comportant des couplages électriques électroniques et mécaniques, et pour lesquels un compromis précision des résultats - temps de calcul est recherché, $[10,18,21-23]$. C'est cette méthode que nous allons ici expliciter, tester et appliquer à l'analyse du comportement d'un système complexe.

Tout d'abord, nous présenterons la méthode des réseaux de perméance, ainsi que la méthode des bond-graphs (graphes de lien) qui y est associée. Ces techniques seront ensuite appliquées à l'étude d'une machine asynchrone à cage rotorique, et les résultats de simulation seront comparés aux résultats expérimentaux. Puis nous étudierons la machine dans un environnement propre à réaliser sa commande vectorielle et nous analyserons les comportements obtenus.

\section{Méthode de modélisation}

1.1. Circuits magnétiques Équivalents. - La représentation des systèmes électromagnétiques par circuit équivalent est basée sur une discrétisation du domaine étudié en tubes de flux : chaque tube est défini par l'ensemble des lignes d'induction s'appuyant sur un contour fermé $\mathcal{C}$ (Fig. 1) et caractérisé par sa perméance $\mathcal{P}$, inverse de la réluctance $\mathcal{R}$. Cette perméance se définit en fonction des caractéristiques géométriques et magnétiques du tube de flux :

$$
\frac{1}{\mathcal{P}}=\mathcal{R}=\int_{A}^{B} \frac{\mathrm{d} \ell}{\mu S_{\mathrm{t}}}=\frac{\varepsilon_{\mathrm{AB}}}{\phi}
$$

$\phi$ est le flux circulant à travers le tube ; $\varepsilon_{\mathrm{AB}}$ est la différence de potentiel magnétique aux bornes du tube de flux. 


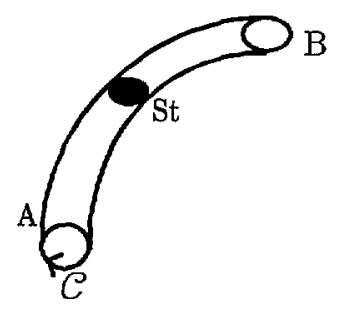

Fig. 1. - Tube de flux.

[Flux tube.]

Le tube de flux constituant l'élément de base de la discrétisation, la perméabilité $\mu$ sera considérée uniforme sur le domaine qu'il définit ; de ce fait, la perméance n'est fonction que de la géométrie du tube de flux et du matériau considéré.

Illustrons la méthode par un exemple simple de conversion électromagnétique : une bobine formée de $n$ spires autour d'un noyau de forme toroïdale alimentée par une source de tension $v(t)$ alternative (Fig. 2a).

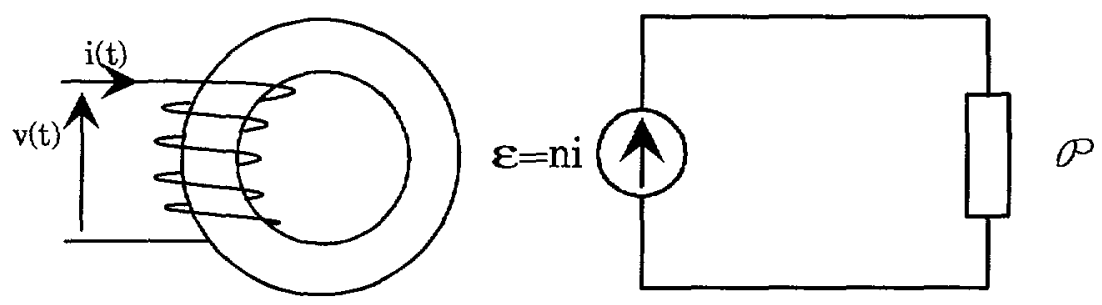

Fig. 2. - a) Bobine à noyau de fer. b) Circuit magnétique équivalent correspondant.

[a) Iron core winding. b) Corresponding magnetic equivalent circuit.]

En négligeant les flux de fuites, nous pouvons caractériser le circuit magnétique de la bobine par une perméance $\mathcal{P}$. D'autre part, l'application du théorème d'Ampère à un contour fermé enlaçant les spires de la bobine donne la relation (2):

$$
\oint \mathbf{H d} \ell=n i=\frac{1}{\mathcal{P}} \phi
$$

Cette équation peut être représentée par le circuit magnétique équivalent de la figure $2 \mathrm{~b}$.

Sur ce schéma, apparaissent les grandeurs magnétiques flux, force magnéto-motrice, et perméance. Pour connaître la valeur de ces grandeurs, à partir de la tension d'alimentation donnée, il est nécessaire de coupler à cette équation magnétique l'équation électrique associée (3) :

$$
v=r_{\imath}+n \frac{\mathrm{d} \phi}{\mathrm{d} t}
$$

où $r$ représente la résistance de la bobine et $v$ sa tension d'alimentation (Fig. 2a). 
À ce niveau, afin de représenter le circuit magnétique et son alimentation de façon unique, de faciliter les couplages et d'introduire les notions de causalité, nous nous sommes appuyés sur la méthode des bond-graphs.

1.2. MÉTHODE DES BOND-GRAPHS [7]. - La technique des "bond-graphs" ou graphes de lien est une méthode de représentation des systèmes physiques basée sur les transferts de puissance. Elle permet de travailler avec une représentation unique dans les différents domaines de la physique (électromagnétisme, thermique, hydraulique...). Cette méthode est basée sur l'expression de la puissance instantanée $p(t)$ échangée entre deux systèmes A et B à l'aide de deux variables générales : l'effort $e$ et le flux $f$ :

$$
p(t)=e(t) f(t)
$$

Le transfert de puissance entre $A$ et $B$ est alors représenté par un lien orienté indiquant le sens du transfert de puissance (Fig. 3).

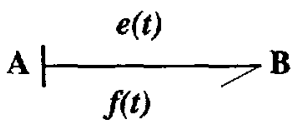

Fig. 3. - Transfert de puissance entre A et $B$.

[Power flow from A to B.]

Sur cette représentation, le trait causal repère l'élément pour lequel l'effort est connu : ce symbole signifiera donc que pour l'élément concerné (ici A), c'est le flux qui se déduit de la relation (4) et, vice versa, l'absence de trait signifiera que l'inconnue est l'effort.

La notion de causalité est ainsi introduite dans la représentation. Par ailleurs, l'imposition des traits causaux sur un schéma bond-graph complexe obéit à des règles strictes, permettant d'établir de proche en proche, les relations de cause à effet entre chaque élément [8]. Ce symbolisme donne ainsi un précieux renseignement quant aux données et aux inconnues du problème et permet l'écriture judicieuse et systématique des équations correspondantes.

Les éléments apparaissant sur un schéma bond-graph sont classés en trois catégories et repérés par les appellations suivantes :

- Les éléments passifs : parmi eux, certains sont le siège de dissipation de puissance et seront notés $R$. Les autres sont des éléments de stockage de l'énergie, ils seront notés $C$ ou $I$ selon leur comportement physique (voir Annexe A).

- Les éléments actifs : ils correspondent à des sources : source d'effort, $S_{\mathrm{e}}$ et source de flux, $S_{\mathrm{f}}$.

- Les éléments de jonction : comme leur nom l'indique, ils matérialisent les liens entre plusieurs éléments appartenant parfois à différents domaines de la physique. On trouve ainsi les "éléments de jonction 0 " (connexion parallèle), les "éléments de jonction 1 " (connexion série), les transformateurs TF et les gyrateurs GY.

Dans notre étude, limitée au comportement dynamique des systèmes électromagnétiques, nous utiliserons un nombre restreint d'éléments. Afin d'illustrer ce formalisme, reprenons 
l'exemple de la bobine. La conversion d'énergie électrique en énergie magnétique se traduit par l'égalité (5) :

$$
[v(t)-r i(t)] i(t) \mathrm{d} t=\varepsilon(t) \mathrm{d} \phi(t)
$$

soit encore en terme de puissance :

$$
E(t) i(t)=\varepsilon(t) \frac{\mathrm{d} \phi(t)}{\mathrm{d} t}
$$

en appelant $E(t)$ la f.e.m. induite aux bornes de la bobine et $\varepsilon(t)$ la force magnétomotrice.

Cette transmission de puissance s'exprime en représentation bond-graph par l'égalité des produits effort-flux pour les deux domaines :

$$
\underbrace{e_{e}(t) f_{e}(t)}_{\text {électrique }}=\underbrace{e_{\mathrm{m}}(t) f_{\mathrm{m}}(t)}_{\text {magnétique }}
$$

En identifiant les termes des équations (6) et (7), il apparaît la correspondance du Tableau I.

Tableau I. - Défnition des efforts et fux dans les domaines électrique et magnétique.

[Bond-graph effort and flux for electromagnetic variables.]

\begin{tabular}{|l|c|c|}
\hline & \multicolumn{2}{|c|}{ domaines } \\
variables bond-graph & électrique & magnétique \\
\hline effort & $E$ & $\varepsilon$ \\
\hline flux & $i$ & $\mathrm{~d} \phi / \mathrm{d} t$ \\
\hline
\end{tabular}

Si cette correspondance apparaît naturellement d'après le bilan de puissance, elle amène néanmoins un résultat inhabituel concernant la variable "flux" bond-graph associée non pas au flux magnétique mais à sa dérivée temporelle [19]. Ceci signifie l'écriture de la relation flux magnétique/force magnéto-motrice (2) sous la forme :

$$
\varepsilon=\frac{1}{\mathcal{P}} \int_{0}^{t} \frac{\mathrm{d} \phi}{\mathrm{d} t} \mathrm{~d} t
$$

soit sous forme bond-graph :

$$
e_{\mathrm{m}}=\frac{1}{C} \int_{0}^{t} f_{\mathrm{m}}(t) \mathrm{d} t
$$

L'élément perméance apparaît donc explicitement comme un élément $C$, de stockage d'énergie magnétique, ce qui est conforme à sa signification physique.

Pour obtenir la représentation bond-graph de la bobine, il suffit de compléter les équations (7) et (9) par celle qui traduit les pertes Joule dans les conducteurs. Le système est alors décrit par le schéma de la figure 4.

Avec :

$$
\begin{array}{ll}
e_{1}=v & e_{2}=r i \\
e_{3}=E & e_{4}=\varepsilon \\
f_{3}=f_{2}=f_{1}=i & f_{4}=\frac{\mathrm{d} \phi}{\mathrm{d} t}
\end{array}
$$




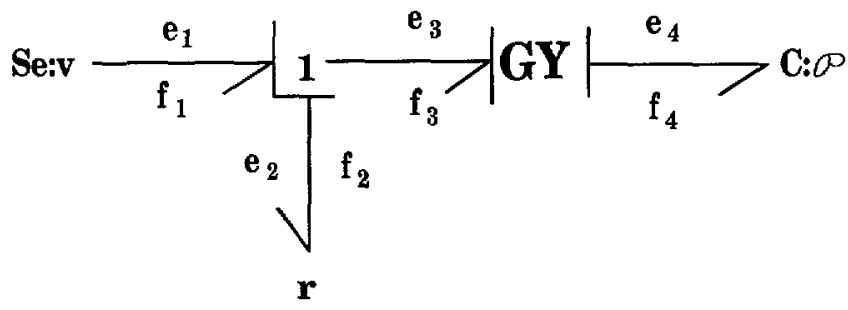

Fig. 4. - Représentation bond-graph de la bobine et de son alimentation.

[Bond-graph scheme of the voltage fed coil.]

Les traits causaux signifient que les inconnues doivent s'écrire en fonction des données selon le système d'équations (10) :

$$
\begin{aligned}
e_{1} & =v & & \text { source d'effort } \\
e_{3} & =e_{1}+e_{2} & & \text { jonction } 1 \\
f_{4} & =\frac{1}{n} e_{3} & & \text { gyrateur } \\
e_{4} & =\frac{1}{\mathcal{P}} \int f_{4} \mathrm{~d} t & & \text { élément } C \\
f_{1} & =\frac{1}{n} e_{4} & & \text { gyrateur } \\
e_{2} & =r f_{1} & & \text { élément } R
\end{aligned}
$$

Sur cet exemple élémentaire, on obtient une représentation unifiée du système à la fois pour ses composantes électrique et magnétique; de plus, la notion de causalité apparaît de façon systématique ce qui permet un ordonnancement correct du système d'équations. Cette propriété, qui semble secondaire pour un tel exemple, trouve son intérêt lorsqu'il s'agit d'étudier des systèmes beaucoup plus lourds. C'est le cas pour l'étude de machines à grand nombre d'encoches, synchrones [18] ou asynchrones [10].

\section{Application à une machine asynchrone à cage}

2.1. Modélisation des armatures ferromagnétiques. - Compte tenu des dimensions habituelles des machines asynchrones, les grandeurs électromagnétiques sont considérées invariantes suivant l'axe de rotation, ce qui revient à négliger les effets d'extrémité (hypothèse 2D).

Les deux armatures rotorique et statorique de la machine sont représentées par un réseau de perméance de topologie identique, tout au moins pour des machines à barres peu profondes. Le choix de cette topologie a été guidé par un souci de simplicité : le réseau ainsi formé constitue le canevas minimum propre à représenter les différents trajets de flux dans la machine [9] (Fig. 5). Il existe d'autres types de schémas magnétiques équivalents plus complexes selon que l'on souhaite tenir compte avec précision de tel ou tel autre phénomène [6].

Pour tenir compte des courants circulant dans les phases statoriques ou induits au rotor, ce réseau de base doit être complété par l'insertion de sources de f.m.m. $\varepsilon_{\mathrm{d}}$ respectant le théorème d'Ampère. Ainsi sur la figure 6, on aura:

$$
\varepsilon d_{\mathrm{j}+1}-\varepsilon d_{\mathrm{j}}=\varepsilon_{\mathrm{j}}=n I_{\mathrm{j}}
$$

avec $n$ le nombre de conducteurs filaires de l'encoche j et $I$ le courant les parcourant. 


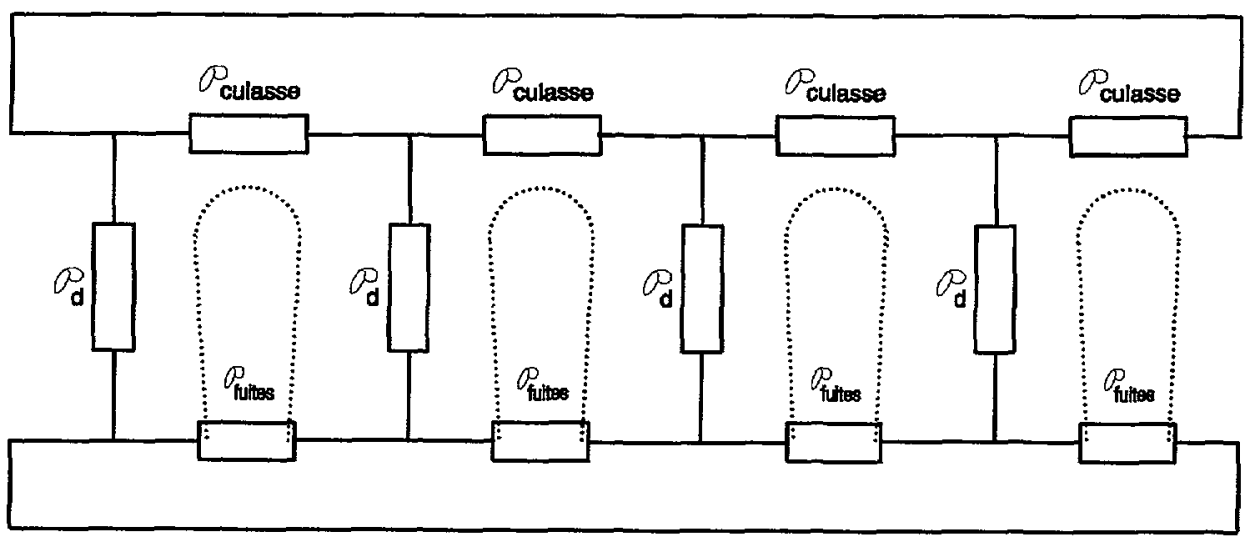

Fig. 5. - Exemple de réseau de perméances au stator.

[Permeance network example for the stator.]

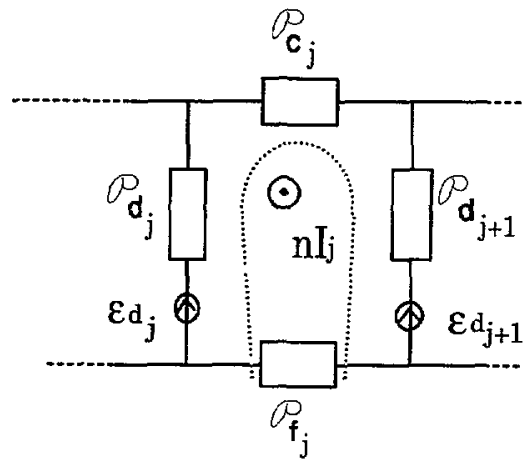

Fig. 6. - Insertion des sources de forces magnétomotrices dans le circuit équivalent.

[Magnetomotive force insertion in the equivalent scheme.]

Cette méthode est appliquée sur les circuits statorique et rotorique ce qui interdit notamment la considération de l'effet pelliculaire. Cette restriction porte peu à conséquence dans l'étude des machines à encoches non profondes ; dans le cas contraire ou pour des machines à double cage, on peut envisager d'affiner le réseau rotorique en ajoutant une perméance de fuite au travers de l'encoche [6].

Le calcul des perméances du réseau ainsi formé est effectué par la méthode des éléments finis : une résolution linéaire en magnétostatique permet effectivement de connaître la différence de potentiel scalaire magnétique $\varepsilon$ et le flux $\phi$. La perméance est alors définie par la relation :

$$
\mathcal{P}=\frac{\phi}{\varepsilon}
$$

Le flux à travers la surface considérée est exprimée par la circulation du potentiel vecteur $\mathbf{A}$ sur le contour de cette surface. Selon l'hypothèse bidimensionnelle, $\phi$ s'écrit (Fig. 7) :

$$
\phi=\left(A_{1}-A_{2}\right) \mathcal{L}
$$

où $\mathcal{L}$ est la longueur utile du dispositif. 


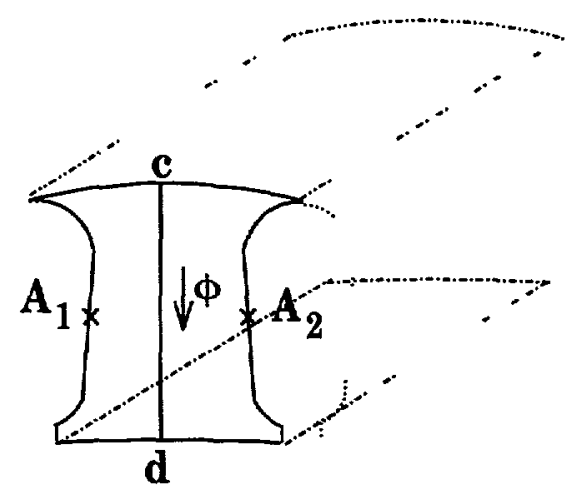

Fig. 7. - Calcul des perméances par la méthode des éléments finis.

[Permeances calculation with finite element method.]

La différence $d u$ potentiel scalaire magnétique $\varepsilon$ pourra être considérée sur une ligne de champ "moyenne".

$$
\varepsilon_{\mathrm{cd}}=\int_{\mathrm{C}}^{\mathrm{D}} \mathbf{H} \mathrm{d} \boldsymbol{\ell}
$$

En pratique, plusieurs calculs de $\varepsilon$ sont effectués selon différentes lignes de champ, et c'est la moyenne des résultats qui est prise en compte.

Cette détermination est obtenue à partir d'un calcul de champ linéaire, les perméances ainsi définies dépendent donc des données géométriques et de la perméabilité maximale du matériau. La saturation magnétique sera prise en compte dans la résolution du système par la variation des perméances selon la valeur du flux. C'est la loi proposée par Marrocco [11] définissant la variation de la perméabilité relative $\mu_{\mathrm{r}}$ en fonction du champ $\mathrm{B}$ qui a été retenue :

$$
\frac{1}{\mu_{\mathrm{r}}}=\varepsilon+(c-\varepsilon) \frac{B^{2 \alpha}}{B^{2 \alpha}+\tau}
$$

Les coefficients $\varepsilon, c$, $\alpha$ et $\tau$ sont établis à partir de la courbe moyenne $B(H)$ du $r$ wériau ; par ailleurs, l'induction $B$ dans le tube de flux sera calculée à partir de la valeur du flux magnétique en considérant la section moyenne du tube. À ce niveau, on réalise alors une approximation d'autant moins grossière que la section du tube de flux considéré est relativement uniforme. En effet, à cause de la non-linéarité magnétique, la perméance équivalente d'un tube est plus proche de celle relative à sa composante de faible section qu'à celle calculée sur sa section moyenne. Selon le degré de précision désiré, le découpage du circuit magnétique en tubes de flux élémentaires doit donc être effectué de façon à pouvoir considérer des sections quasi-uniformes. Une amélioration possible pour la prise en compte de la saturation consisterait également à introduire directement dans la résolution les caractéristiques $\mathcal{P}(\varphi)$ (perméances en fonction du flux) elles-mêmes calculées par éléments finis. Cette démarche alourdirait cependant la phase préparatoire de la méthode, aussi ne faut-il l'envisager que dans le cas où les disparités de sections sont importantes et lorsque le cahier des charges, concernant les résultats, l'exige. 

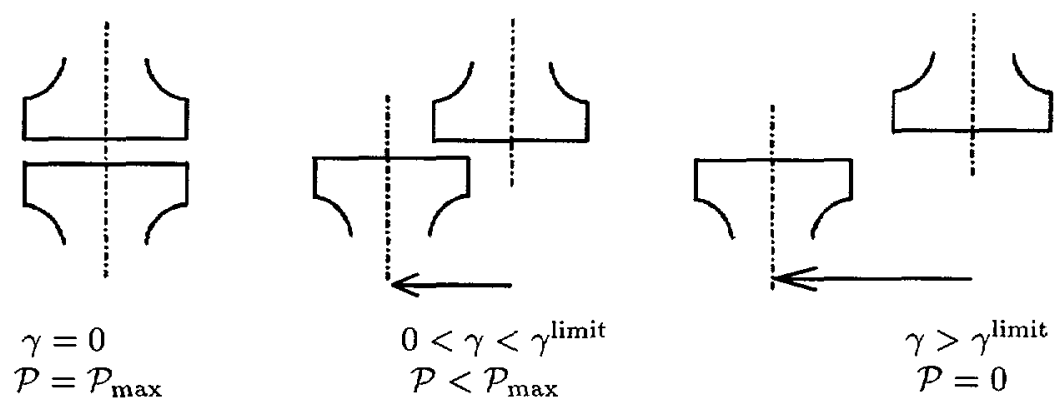

Fig. 8. - Positions relatives des dents statoriques et rotoriques.

[Relative positions of rotor and stator teeth.]

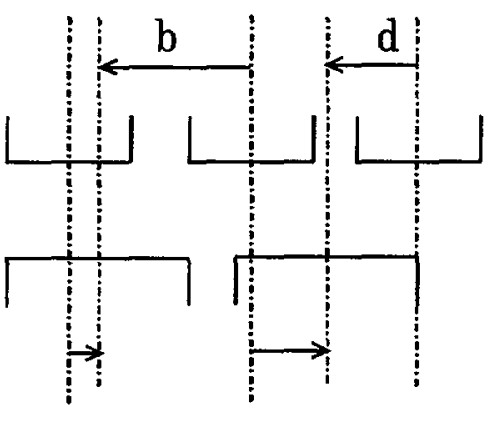

a

c

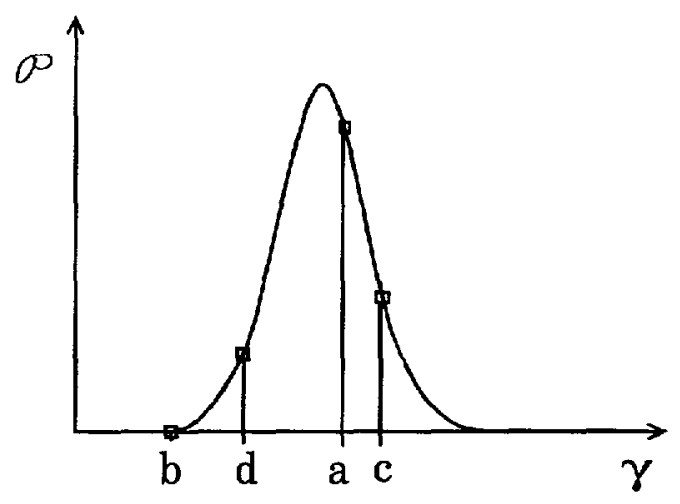

Fig. 9. - Détermination des perméances d'entrefer.

[Air-gap permeances determination.]

2.2. ModélisATION DE L'ENTREFER. - Conformément à notre démarche, pour modéliser l'entrefer, nous introduisons un élément perméance entre chaque dent statorique $i$ et chaque dent rotorique $j$. Au cours de la rotation, la perméance $\mathcal{P}_{\imath \jmath}$ augmente lorsque les dents $i$ et $j$ se rapprochent, passe par une valeur maximale lorsque les deux sont face à face puis diminue lorsqu'elles s'éloignent l'une de l'autre. On définit alors $\gamma_{\imath \jmath}$ limit tel que $\mathcal{P}_{\imath \jmath}$ soit nulle lorsque les axes des deux dents sont séparés d'un angle supérieur à $\gamma_{\text {limit }}$ (Fig. 8).

Chaque perméance $\mathcal{P}_{2 j}$ aura une évolution identique selon l'angle $\gamma_{\imath_{3}}$, et pour calculer les valeurs de cette courbe, nous nous sommes appuyés comme précédemment sur les résultats d'un calcul par éléments finis. Compte tenu de la répétition des motifs géométriques au stator comme au rotor, une seule position permet de calculer plusieurs points de la courbe $\mathcal{P}_{\imath \jmath}=f\left(\gamma_{\imath \jmath}\right)$ (Fig. 9). Cette remarque permet de limiter à deux ou trois le nombre de calculs numériques nécessaires à la définition de la courbe.

La périodicité angulaire de la fonction "perméance d'entrefer" nous a ensuite guidés vers une interpolation trigonométrique pour la résolution numérique :

$$
\mathcal{P}_{2 \jmath}\left(\gamma_{\imath \jmath}\right)=\frac{a_{0}}{2}+\sum_{k=1}^{n}\left[a_{k} \cos \left(p k \gamma_{\imath \jmath}\right)-b_{k} \sin \left(p k \gamma_{\imath \jmath}\right)\right]
$$




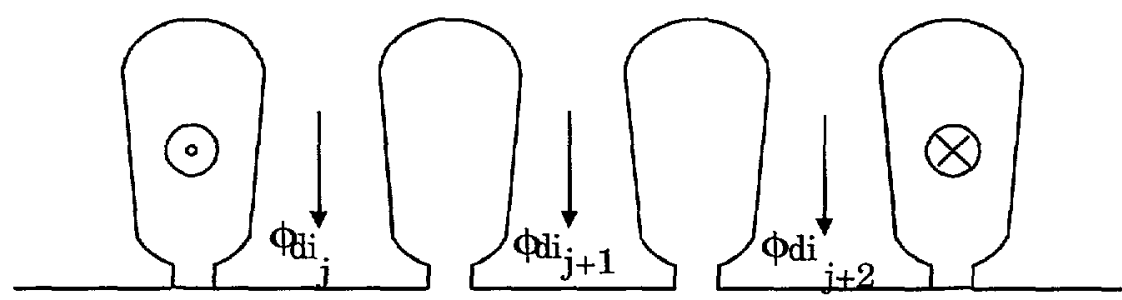

Fig. 10. - Flux relatifs à une phase statorique.

[Stator phasis fluxes.]

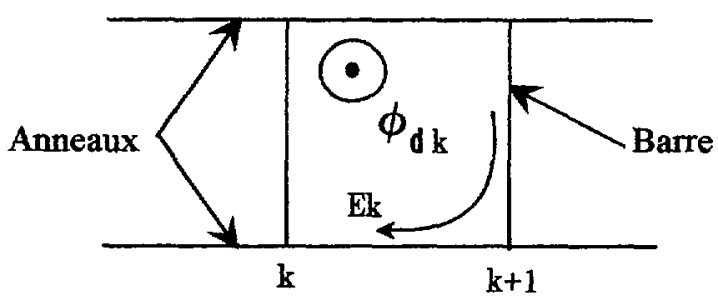

Fig. 11. - F.e.m. induite sur une phase rotorique.

[Induced electromotive force on a rotor phasis.]

À noter que cette écriture est particulièrement bien adaptée à la prise en compte de l'inclinaison des encoches [9].

2.3. Couplages Électriques. - Dans le cas particulier de la machine asynchrone, deux couplages électriques sont nécessaires, décrivant respectivement l'alimentation des bobines statoriques et le court-circuit des barres rotoriques.

Ces couplages nécessitent la connaissance des forces électromotrices induites dans chaque phase. Celles-ci sont exprimées en fonction du flux magnétique total capté par le circuit en question, lui-même connu dans notre modélisation, grâce aux flux à travers chaque dent des armatures.

2.3.1. F.e.m. induites. - Ainsi au stator, pour une phase $i$ comportant $n$ spires par pôle et concentrée dans deux encoches, la f.e.m. s'écrit (Fig. 10) :

$$
E_{\imath}=p n\left[\frac{\mathrm{d} \phi_{\mathrm{d} \imath_{3}}}{\mathrm{~d} t}+\frac{\mathrm{d} \phi_{\mathrm{d} \imath_{3+1}}}{\mathrm{~d} t}+\frac{\mathrm{d} \phi_{\mathrm{d} \imath_{j+2}}}{\mathrm{~d} t}\right]
$$

avec $p$ le nombre de paires de pôles.

Au rotor, on définit une phase par l'association de deux barres voisines et des portions d'anneau qui les relient. La f.e.m. $E_{k}$ induite sur cette maille s'écrit également en fonction du flux $\phi_{\mathrm{d} k}$ dans la dent correspondante (Fig. 11) :

$$
E_{k}=\frac{\mathrm{d} \phi_{\mathrm{d} k}}{\mathrm{~d} t}
$$




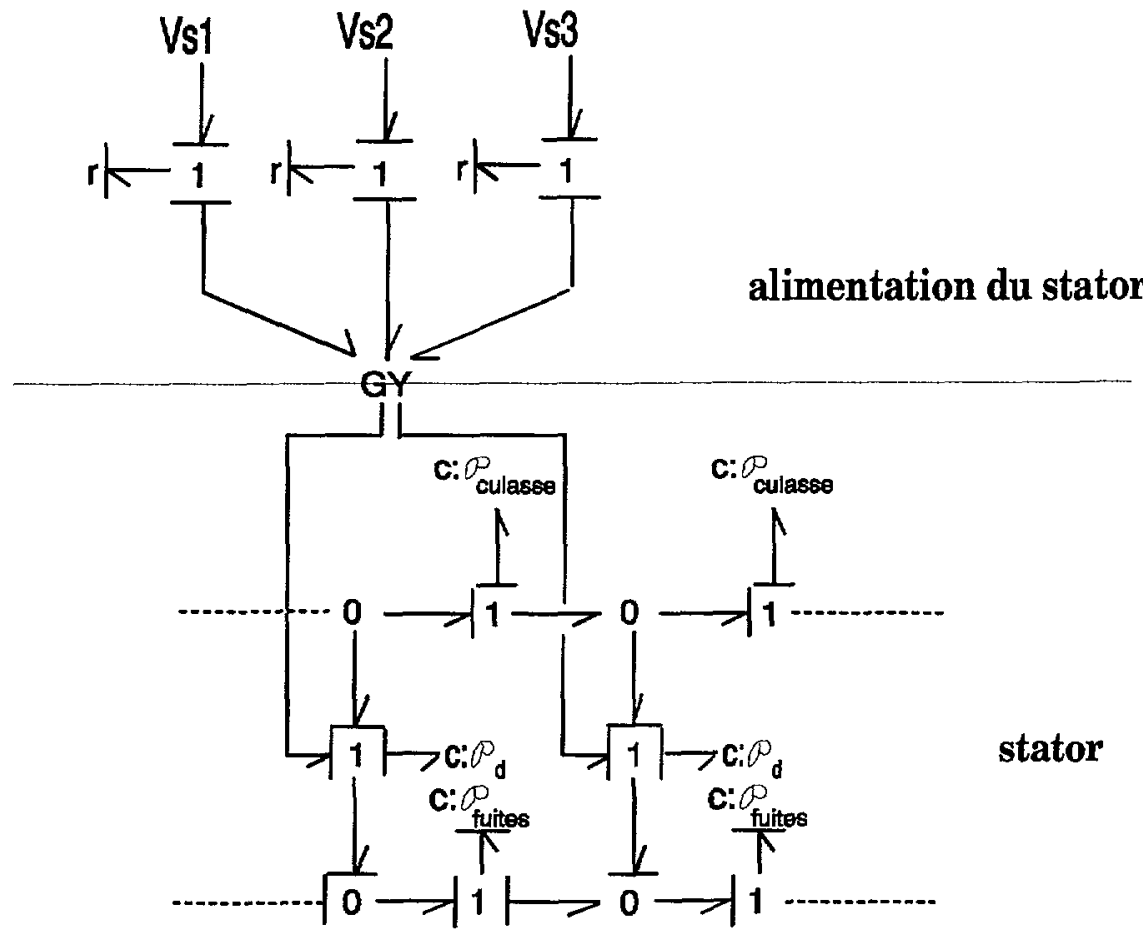

Fig. 12. - Représentation du schéma bond-graph d'un motif magnétique statorique élémentaire.

[Bond-graph scheme of a stator elementary magnetic circuit.]

2.3.2. Équations électromagnétiques. - Les équations électriques relatives à chaque phase s'écrivent de la façon suivante :

$$
v_{k}=r_{k} i_{k}+E_{k}
$$

où $r_{k}$ représente la résistance de la phase $k$, et $v_{k}$ la tension d'alimentation. Dans le cas du circuit rotorique, les tensions $v_{k}$ sont nulles, les résistances $r_{k}$ sont déterminées par la résistance des barres rotoriques et par celle de l'anneau de court-circuit. On notera $R_{\mathrm{b}}$ eq la résistance totale de chaque maille rotorique.

En appliquant la méthode des bond-graphs selon la démarche exposée précédemment, on obtient une représentation unique pour les parties électriques et magnétiques au stator (Fig. 12) comme au rotor (Fig. 13). L'écriture des équations relatives à ces schémas (et à leur association pour décrire complètement le domaine d'étude) est toujours guidée par l'imposition des traits causaux.

Le couplage électromagnétique peut être décrit par le formalisme matriciel suivant, qui s'appuie sur les équations (11), (12), (17), (18) et (19) :

$$
\left[\begin{array}{ll}
{\left[\mathcal{P}^{-1}\right]} & {[n]} \\
{\left[n \frac{\mathrm{d}}{\mathrm{d} t}\right]} & {[r]}
\end{array}\right]\left[\begin{array}{l}
{[\phi]} \\
{[i]}
\end{array}\right]=\left[\begin{array}{l}
{[0]} \\
{[v]}
\end{array}\right]
$$

Les matrices et vecteurs qui apparaissent dans ce système sont relatifs au réseau complet, et font intervenir des grandeurs statoriques, rotoriques et d'entrefer. Ainsi $\left[\mathcal{P}^{-1}\right]$ est la matrice 


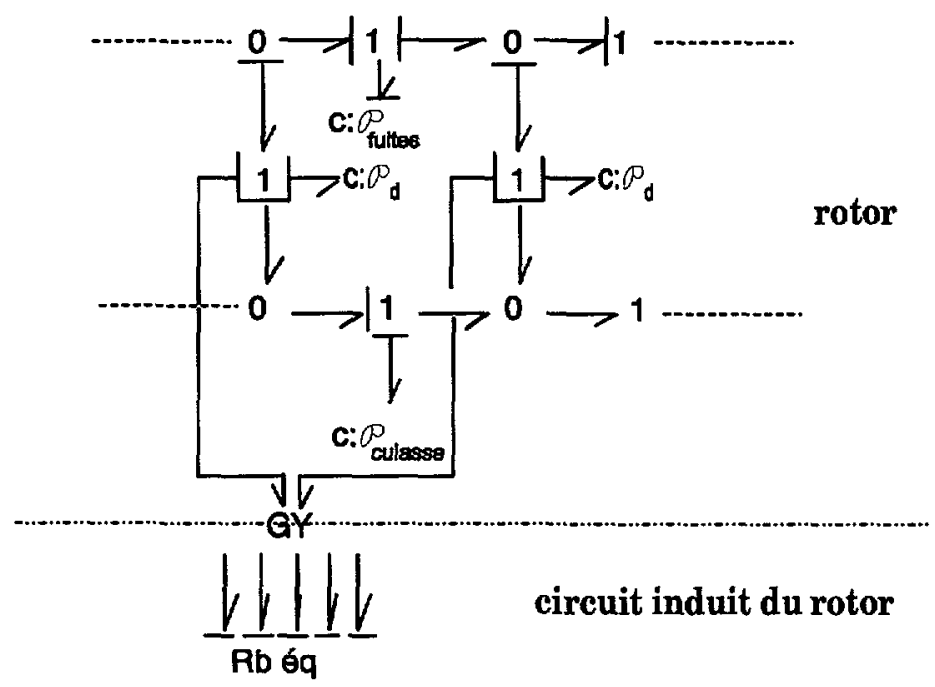

Fig. 13. - Schéma bond-graph du circuit électromagnétique rotorique élémentaire.

[Bond-graph scheme of rotor elementary electromagnetic circuit.]

des réluctances du réseau, $[r]$ la matrice des résistances statoriques, de barres et d'anneau, $[\phi]$ le vecteur des flux magnétiques dans chaque branche du réseau, [i] le vecteur des courants statoriques et rotoriques, et $[v]$ le vecteur des tensions statoriques et rotoriques.

2.4. Couplage mÉCAnique. - La méthode de simulation envisagée doit pouvoir tenir compte des phénomènes transitoires en vitesse, aussi est-il nécessaire de considérer l'équation mécanique du système :

$$
J \frac{\mathrm{d} \Omega}{\mathrm{d} t}=C_{\mathrm{em}}-f \Omega-C_{\mathrm{res}}
$$

$J$ représente le moment d'inertie total ramenée à l'arbre moteur, $\Omega$ la vitesse de rotation mécanique, $C_{\mathrm{em}}$, le couple électromagnétique, $f$ le coefficient de frottements visqueux et $C_{\text {res }}$ le couple résistant sur l'arbre. Le couplage mécanique consiste à exprimer le couple $C_{\text {em }}$ en fonction des variables magnétiques de la machine. Sur la base d'un bilan énergétique, le couple électromagnétique s'exprime en fonction de l'énergie magnétique [12] :

$$
C_{\mathrm{em}}=-\left.\frac{\partial W_{\mathrm{mag}}}{\partial \theta}\right|_{\imath=\mathrm{cte}}
$$

En prenant pour hypothèse que les perméances d'entrefer ne dépendent que de $\theta$, la position relative stator/rotor, on arrive à l'expression suivante pour $C_{\mathrm{em}}[9,13]$ :

$$
C_{\mathrm{em}}=\frac{1}{2} \sum_{\imath=1}^{n_{\mathrm{s}}} \sum_{\jmath=1}^{n_{\mathrm{r}}} \frac{\mathrm{d} \mathcal{P}_{\imath \jmath}}{\mathrm{d} \theta} \varepsilon_{\imath \jmath}^{2}
$$

avec $n_{\mathrm{s}}$ le nombre de dents statoriques, $n_{\mathrm{r}}$ le nombre de dents rotoriques et $\varepsilon_{\imath \jmath}$ la différence de potentiel scalaire magnétique entre les dents $i$ statorique et $j$ rotorique.

Le couplage électro-mécanique se concrétise donc par les équations (21) et (23), et fait intervenir les grandeurs électromagnétiques caractéristiques de l'entrefer. 
2.5. RÉSOLUTION NUMÉRIQUE. - La simulation de la machine en régime dynamique nécessite la résolution du système d'équations (20) (21) et (23). La prise en compte de la saturation magnétique selon la loi (15) nécessite une résolution itérative. La technique des bond-graphs donne une représentation unique du processus électromécanique ainsi modélisé (Fig. 14) ; l'imposition méthodique des traits causaux permet de vérifier la description correcte du système. L'ordonnancement des équations s'en déduit également, selon la démarche exposée au paragraphe 1.2 .

Pratiquement, cette simulation a été effectuée par le logiciel de résolution d'équations algébrodifférentielles Allan-Neptunix ${ }^{\circledR}$, adapté au traitement de tels problèmes et utilisant les propriétés de la méthode bond-graph [14].

\section{Modélisation d'une machine asynchrone à cage}

3.1. PRÉSEntation du Système. - La machine étudiée est une machine asynchrone tétrapolaire à cage rotorique de puissance égale à $3 \mathrm{~kW}$ sous $220 \mathrm{~V} / 50 \mathrm{~Hz}$. Elle compte 36 encoches au stator et 26 barres de cuivre au rotor ; le bobinage statorique est distribué dans trois encoches par pôle par phase. La figure 15 représente la section étudiée ainsi qu'un tracé de lignes de champ obtenu pour un fonctionnement à vide.

Des essais expérimentaux classiques ont permis la caractérisation de la machine, les paramètres inductifs ont par ailleurs été déterminés également par calcul de champ ; les résultats sont regroupés et comparés en annexe $\mathrm{B}$.

3.2. Méthodes de simulation. - Afin de tester la méthode proposée tant au niveau de la précision qu'à celui du temps de calcul, nous avons comparé ses résultats avec ceux issus de méthodes de modélisation plus classiquement utilisées : la méthode analytique dite "de Park" d'une part, la méthode des éléments finis couplés aux équations de circuit d'autre part.

La méthode analytique repose sur les équations électriques caractérisant une machine diphasée/diphasée équivalente; ces équations sont écrites dans un repère quelconque, que nous choisissons ici lié au stator par souci de simplicité. En considérant par exemple comme variables d'état les courants statoriques et les composantes du flux rotorique, on obtient le système suivant :

$$
\frac{\mathrm{d}[X]}{\mathrm{d} t}=[A][X]+[B][u]
$$

avec

$$
\begin{gathered}
A=\left[\begin{array}{cccc}
-\frac{1}{\sigma T_{\mathrm{s}}}-\frac{1-\sigma}{\sigma T_{\mathrm{r}}} & 0 & \frac{1-\sigma}{\sigma T_{\mathrm{r}} M_{\mathrm{sr}}} & \frac{1-\sigma}{\sigma M_{\mathrm{sr}}} p \Omega \\
0 & -\frac{1}{\sigma T_{\mathrm{s}}}-\frac{1-\sigma}{\sigma T_{\mathrm{r}}} & -\frac{1-\sigma}{\sigma M_{\mathrm{sr}}} p \Omega & \frac{1-\sigma}{\sigma T_{\mathrm{r}} M_{\mathrm{sr}}} \\
\frac{M_{\mathrm{sr}}}{T_{\mathrm{r}}} & 0 & -\frac{1}{T_{\mathrm{r}}} & -p \Omega \\
0 & \frac{M_{\mathrm{sr}}}{T_{\mathrm{r}}} & p \Omega & -\frac{1}{T_{\mathrm{r}}}
\end{array}\right] \\
u=\left[\begin{array}{l}
V d_{\mathrm{s}} \\
V q_{\mathrm{s}}
\end{array}\right] \text { et }[B]=\frac{1}{\sigma L_{\mathrm{cs}}}\left[\begin{array}{ll}
0 & 0 \\
0 & 0 \\
1 & 0 \\
0 & 1
\end{array}\right] \quad[X]=\left[\begin{array}{l}
I d_{\mathrm{s}} \\
I q_{\mathrm{s}} \\
\phi d_{\mathrm{r}} \\
\phi q_{\mathrm{r}}
\end{array}\right]
\end{gathered}
$$

Dans ces équations, apparaissent des paramètres résistifs et inductifs définis en annexe $B$, qui sont considérés constants et obtenus à partir des essais expérimentaux. Le vecteur [u] 


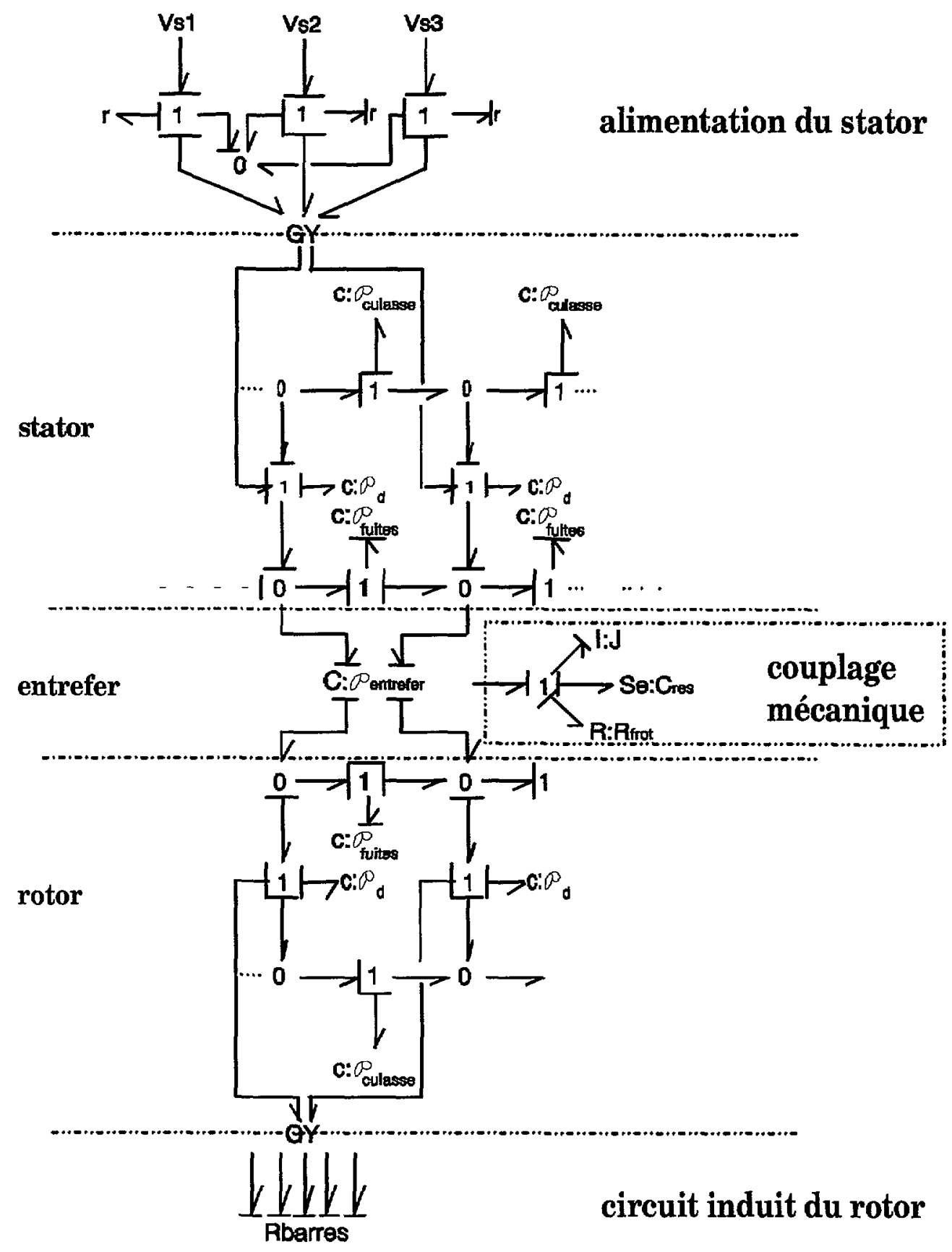

Fig. 14. - Schéma bond-graph complet de la machine et de son alimentation.

[Complete bond-graph scheme of the voltage fed induction machine.] 


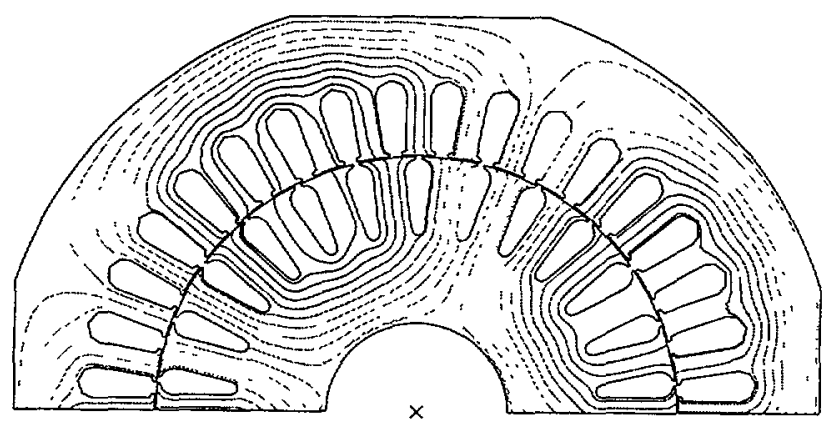

Fig. 15. - Section étudiée correspondant à une paire de pôles.

[Studied area corresponding to a pole pair.]

est constitué des tensions d'alimentation statorique. Associé à l'équation mécanique (21), ce système permet la simulation de la machine alimentée en tension, en régime dynamique.

La méthode des éléments finis utilisée est une méthode $2 \mathrm{D}$ assurant le couplage avec les équations de circuit et travaillant en pas à pas dans le temps [15]. Les éléments finis sont d'ordre deux et la prise en compte du mouvement est assurée par un macro-élément dans l'entrefer ; la saturation magnétique est introduite dans la résolution selon la loi (15). Compte tenu du nombre de barres au rotor, il est nécessaire de mailler une demi-machine soit un total de 7172 nœuds.

3.3. COMPARAISON DES RÉSultats. - Afin de valider notre méthode de modélisation, nous avons tout d'abord procédé à l'étude de la machine alimentée en tension sinusoïdale et à vitesse fixe. Dans un premier temps, le moteur à vide est soumis à un système de tension de valeur efficace réduite à $20 \%$ de la tension nominale. Les formes d'onde obtenues sur le courant statorique montrent alors des courbes sinusoïdales équilibrées, conformes à un état non saturé de la machine (Fig. 16). Les résultats des différentes simulations concordent avec les résultats expérimentaux ; la méthode analytique, dont les paramètres ont été identifiés expérimentalement dans les conditions magnétiques correspondant à cet essai donne également des formes d'onde proches des courants réels.

Dans un deuxième temps, afin de mettre en évidence l'effet de saturation magnétique, la machine, dans des conditions de charge identiques, est alimentée en tension nominale. Expérimentalement, il apparaît un déséquilibre sur les courants statoriques, ainsi qu'une déformation caractéristique des formes d'ondes (Fig. 17) ; l'analyse spectrale révèle d'ailleurs la présence d'harmoniques 3 et 5 du fondamental. Ce déséquilibre s'explique par la forme non régulière de la machine (Fig. 15) : les troncatures effectuées sur l'extérieur de l'armature statorique spécifient chaque phase l'une par rapport à l'autre, en particulier pour des fonctionnements en saturation. L'étude par la méthode des éléments finis d'une machine semblable en tous points mais possédant une armature statorique cylindrique a d'ailleurs prouvé l'origine du déséquilibre : dans ce cas, des deux premiers harmoniques impairs, seul l'harmonique 5 subsiste, du fait de la saturation magnétique [9].

La simulation de ce point de fonctionnement a été effectuée par les trois méthodes (Figs. 1820). La méthode des éléments finis tient compte par nature de la géométrie réelle du système ; la méthode des réseaux de perméance, dans une moindre mesure, en tient compte également, grâce à des perméances de culasse statorique dont la valeur varie selon que l'on décrit la partie 


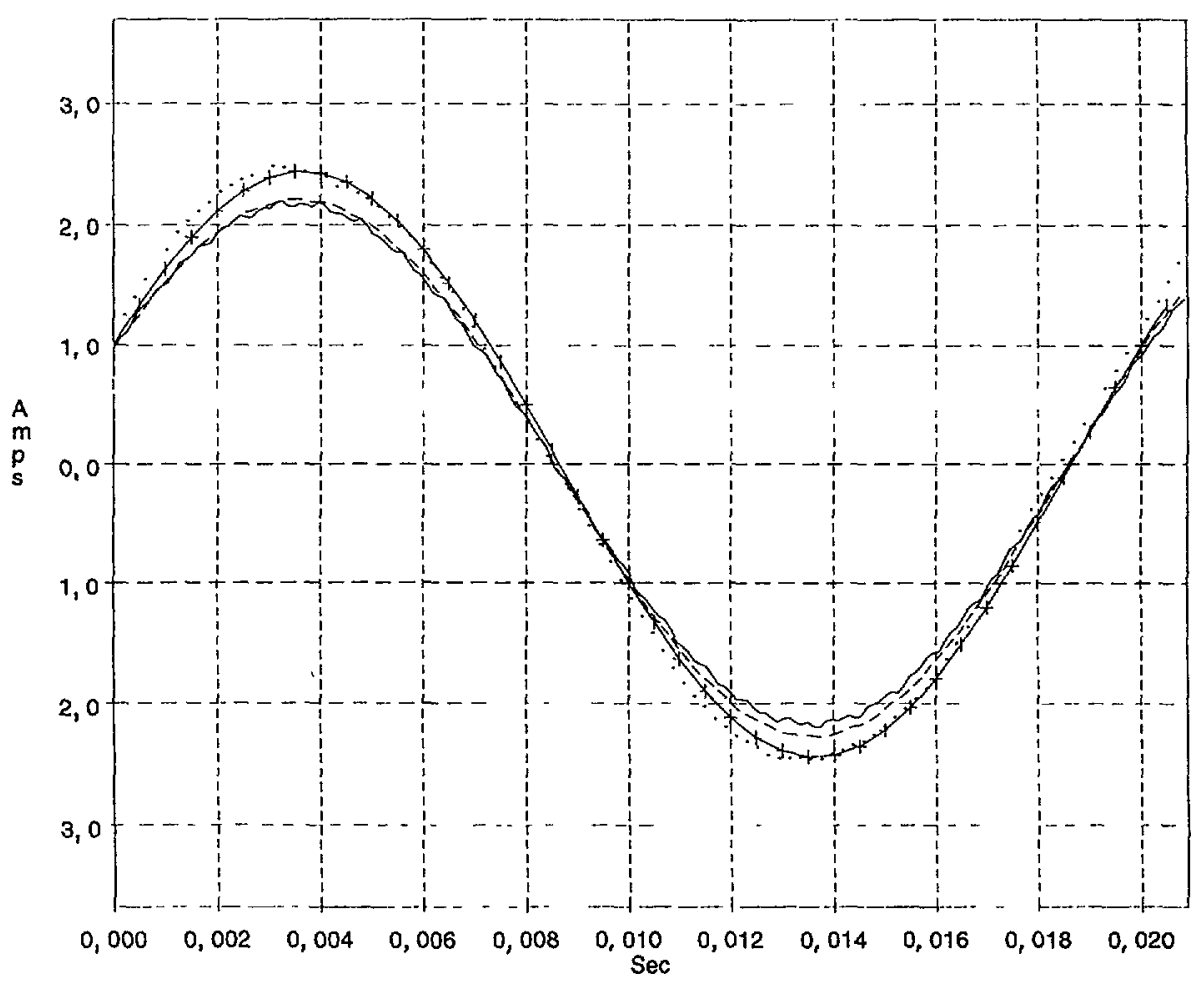

Fig. 16. - Courants statoriques absorbés à vide sous tension réduite : (..) expérimentaux, $(+++)$ méthode analytique, $(--)$ méthode des réseaux de perméances, $(-)$ méthode des éléments finis.

[Stator currents at no-load for reduced voltage fed machine: (. . ) experimental results, $(+H)$ analytical method, (- - ) permeance network method, ( - ) finite element method.]

cylindrique de la machine ou la partie tronquée. La méthode analytique (Fig. 20) par contre ne fait pas intervenir cette différence, on peut noter simplement que là encore les paramètres utilisés ont été identifiés expérimentalement dans des conditions magnétiques correspondantes. L'analyse de ces résultats amène aux remarques suivantes. On constate tout d'abord que seules les deux méthodes numériques (éléments finis et réseaux de perméances) peuvent prendre en compte des phénomènes locaux tels l'irrégularité géométrique du stator et la saturation magnétique. Également, la méthode des éléments finis donne des allures de courant, (en particulier pour la phase a), sensiblement différentes des comportements expérimentaux. Ceci peut s'expliquer par l'approximation bidimensionnelle et par l'imprécision de la caractérisation magnétique du matériau. L'écart enregistré entre les résultats issus des modélisations par éléments finis et réseau de perméances (notamment $10 \%$ sur l'amplitude du courant $I_{b}$ ) est par contre d'une autre nature puisque les deux simulations reposent sur des données parfaitement identiques, tant du point de vue géométrique que magnétique. Cet effet est imputable, d'une part à la discrétisation sommaire de la géométrie du domaine d'étude qui, dans le cas des schémas magnétiques équivalents, tend à déformer la distribution spatiale du flux. D'autre part, compte tenu de la géométrie des dents de la machine, la remarque énoncée au paragraphe 2.1. souligne une sur-estimation de la valeur de la perméance en régime non linéaire. Ces deux facteurs contribuent à la différence de distribution spatiale de flux dans les dents pour les deux 


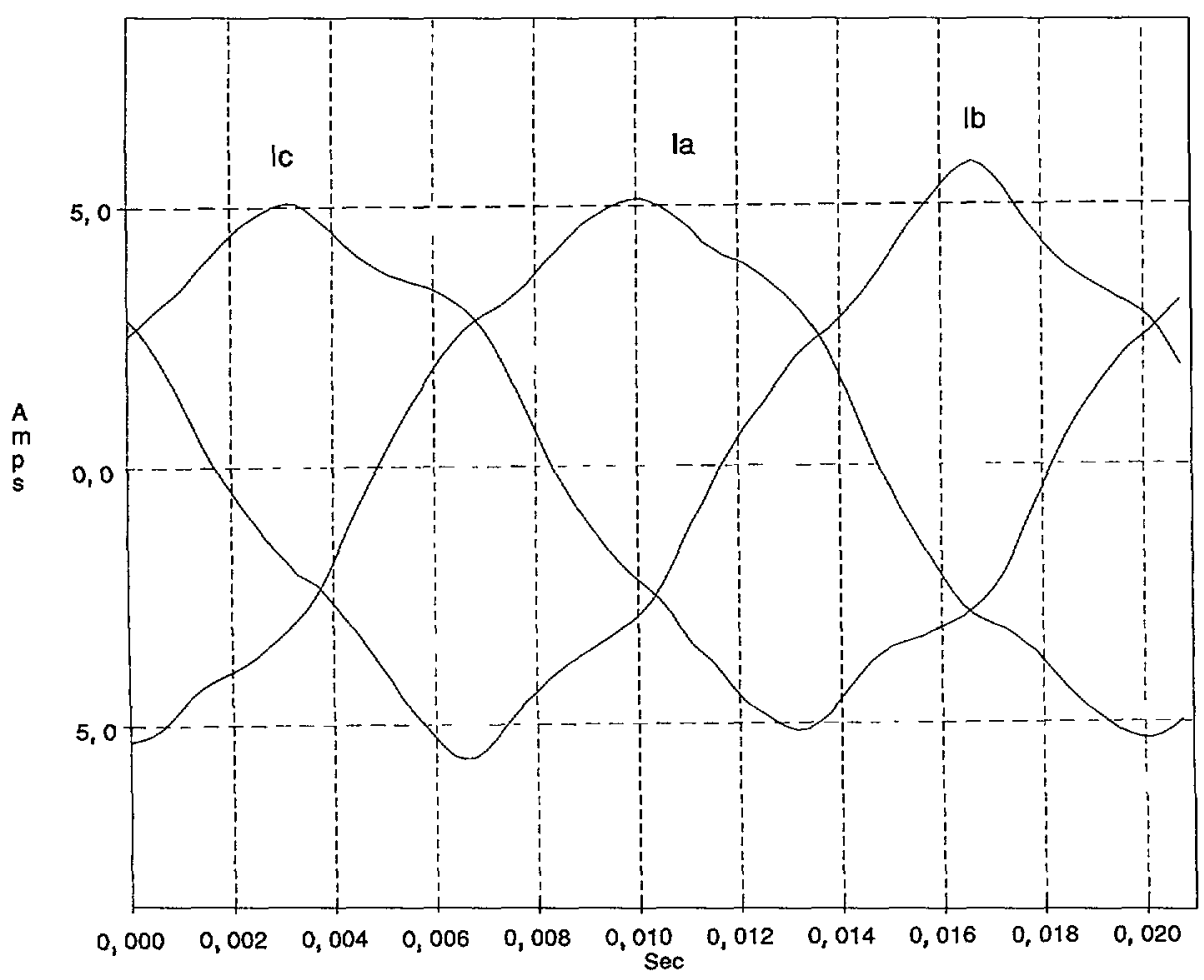

Fig. 17. - Courants statoriques absorbés à vide sous tension nominale : résultats expérimentaux.

[Stator currents at no-load for nominal voltage fed machine: experimental results.]

méthodes et à l'écart de courant qui s'en suit. Il est clair que la réduction drastique du temps de calcul (il existe un rapport 30 entre la méthode des éléments finis et celle des réseaux de perméance au profit de cette dernière) s'effectue au détriment de la précision des résultats. Néanmoins, ceux-ci restent tout à fait corrects et suffisamment satisfaisants pour l'étude de systèmes complexes.

Un essai à rotor bloqué sous tension réduite a également été réalisé. Le matériau magnétique n'étant pas saturé, les trois courants statoriques sont cette fois sinusoïdaux ; les amplitudes sont similaires pour les différentes méthodes et coïncident avec les résultats expérimentaux, ce qui montre, entre autres, pour la méthode proposée, la bonne prise en compte du couplage électrique rotorique (Fig. 21).

Ces résultats, obtenus en régime permanent sinusoïdal, ont été complétés par des tests en régime dynamique : en particulier un démarrage sous tension nominale à vide a été simulé (Fig. 22a). À titre comparatif, le résultat issu de la simulation analytique est présenté figure $22 \mathrm{~b}$. On constate des évolutions du couple et de la vitesse similaires pour les deux méthodes ; néanmoins, la prise en compte de la géométrie réelle et de la denture fait apparaître des harmoniques haute fréquence sur le couple obtenu par la méthode des réseaux de perméances, phénomène tout à fait absent pour la simulation analytique. La prise en compte de la saturation magnétique par la méthode proposée provoque également un écart sur l'amplitude des oscillations transitoires du couple électromagnétique [16]. 


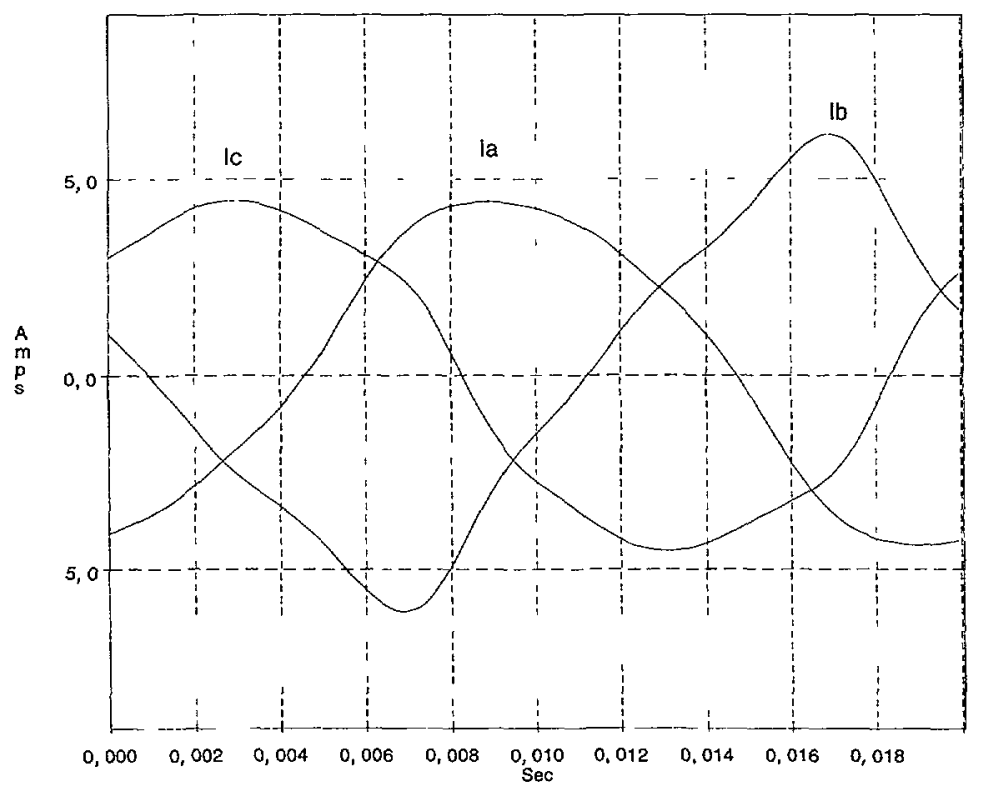

Fig. 18. - Courants statoriques absorbés à vide sous tension nominale : simulation par la méthode des éléments finis.

[Stator currents at no-load for nominal voltage fed machine: finite element method computation.]

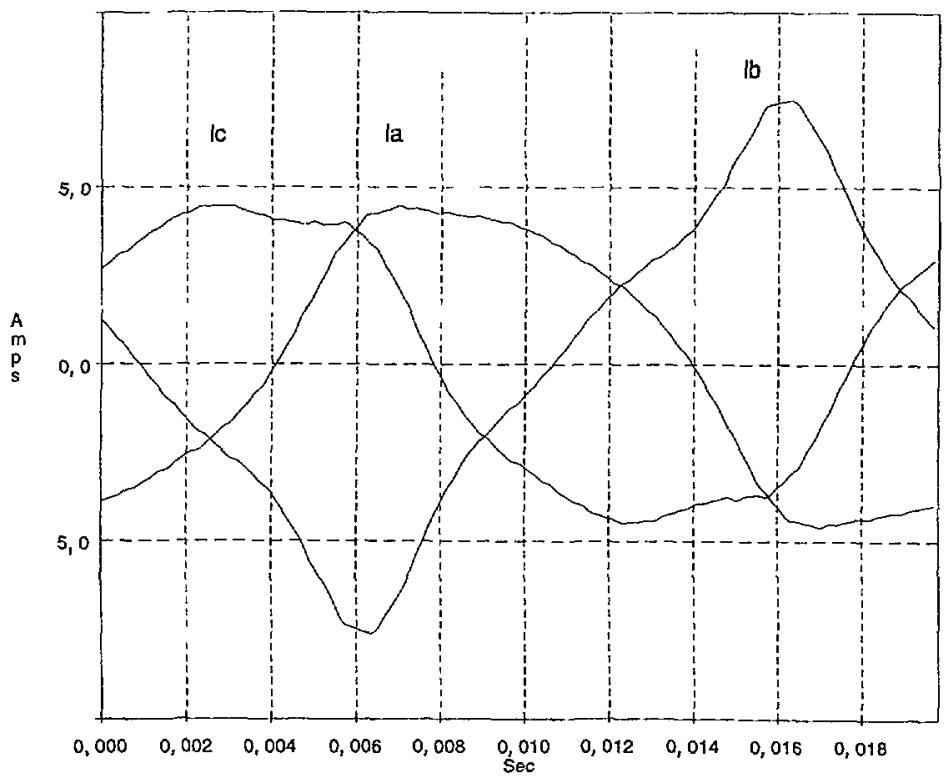

Fig. 19. - Courants statoriques absorbés à vide sous tension nominale : simulation par la méthode des réseaux de perméances.

[Stator currents at no-load for nominal voltage fed machine: permeance network method computation.] 


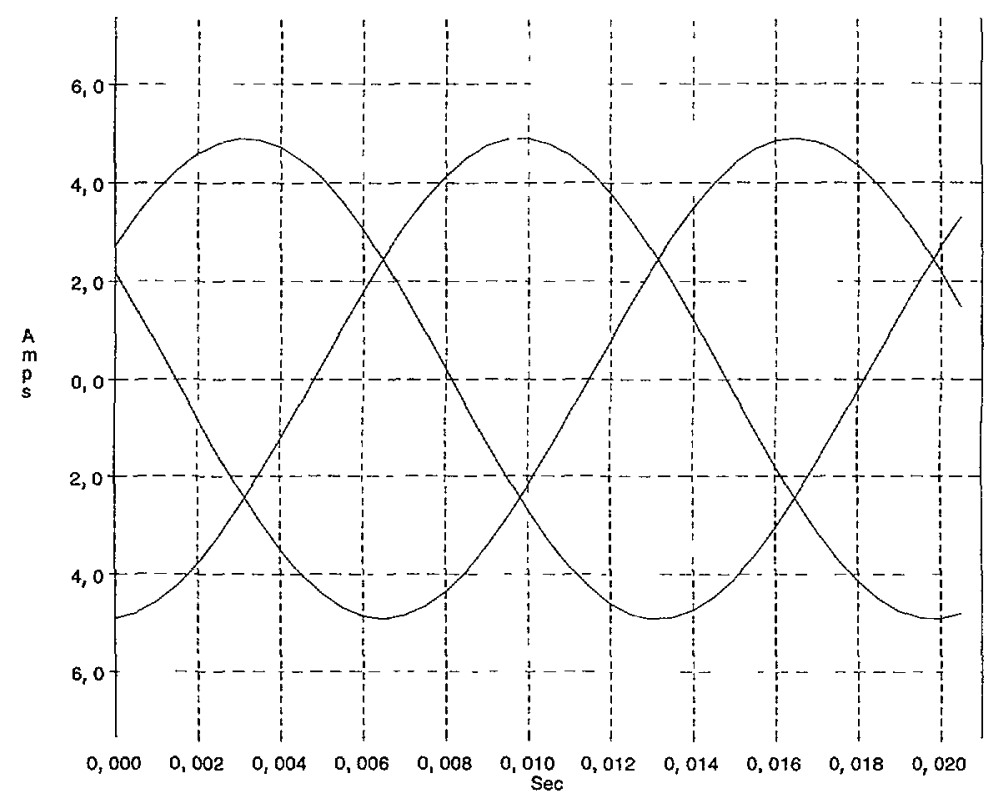

Fig. 20. - Courants statoriques absorbés à vide sous tension nominale : simulation par la méthode analytique.

[Stator currents at no-load for nominal voltage fed machine: analytical method results.]

Ces résultats concernant l'étude de la machine seule ainsi que le bon compromis précision/temps de calcul présenté par la méthode des réseaux de perméance, permet d'envisager la simulation de systèmes complexes. Dans cette optique, nous avons simulé le comportement de la machine commandée vectoriellement.

L'algorithme de commande repose sur une représentation analytique de la machine basée sur le système (24), dans le repère du flux rotorique [17]. Les contrôles des flux, couple, vitesse sont organisés en boucles imbriquées, et l'onduleur de tension qui alimente la machine a pour rôle d'imposer la valeur moyenne des tensions générées par les asservissements de courant. Cet onduleur, destiné expérimentalement à fonctionner en M.L.I., est simulé par une fonction gain, imposant à chaque instant d'échantillonnage ces tensions de référence. D'autre part, le flux rotorique, non mesuré expérimentalement, est reconstitué pour la synthèse de la commande à partir des équations (24). À noter que cette fois, les paramètres inductifs utilisés dans ces équations sont ceux déterminés par la méthode des éléments finis, à partir des calculs ayant eux-mêmes permis la détermination des perméances du réseau (Annexe B).

Afin d'étudier le comportement magnétique de la machine dans ces conditions, le flux est également reconstitué à partir des flux dans chaque dent rotorique, grâce aux équations du réseau de perméances. Le but de cette démarche est d'analyser les réactions de la machine lorsqu'elle est soumise à une commande élaborée à partir d'un modèle approximatif (modèle de Park). Nous avons alors simulé l'excitation magnétique de la machine puis sa réponse indicielle en vitesse (Fig. 23). Au regard du flux reconstitué analytiquement et du flux obtenu par la méthode des réseaux de perméances (que nous appellerons abusivement "\#lux réel"), on peut remarquer deux effets. Tout d'abord, il est manifeste que le phénomène de saturation magnétique, pris en compte dans le modèle de machine mais non dans celui de sa commande, 


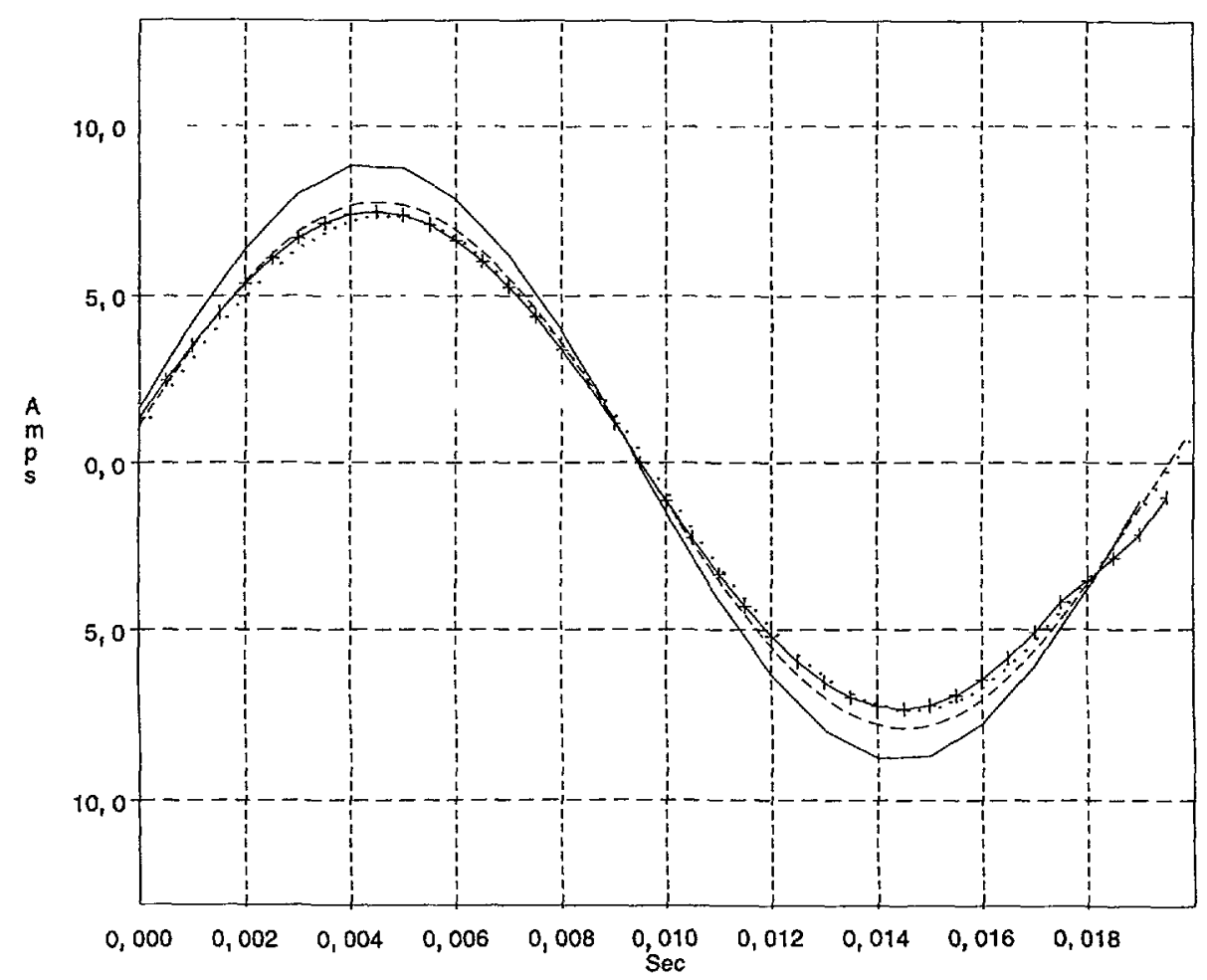

Fig. 21. - Courants statoriques absorbés à rotor bloqué sous tension réduite : (..) expérimentaux, (+1) méthode analytique, $(---)$ méthode des réseaux de perméances, $(-)$ méthode des éléments finis.

[Stator currents at looked-rotor for reduced voltage fed machine: $(.$.$) experimental results, (+++)$ analytical method, (- - ) permeance network method, ( - ) finite element method.]

limite la croissance du flux lors de la phase d'excitation. Cet aspect n'est par contre pas visible sur le flux reconstitué, le réglage du système ne peut donc en tenir compte. De plus, quand la vitesse de rotation augmente, on constate une fluctuation du flux "réel" alors que le flux reconstitué y reste insensible. Ce couplage entre les voies $d$ et $q$ de la machine s'explique par une erreur d'orientation du repère. Cette erreur peut trouver son origine dans de multiples causes. En l'occurrence, ici, la mauvaise reconstitution du module du flux en représente une majeure.

L'exemple de cet essai montre qu'une modélisation plus fine de la machine mais néanmoins suffisamment légère, permet l'analyse des lois de commande, et peut naturellement amener par la suite à la synthèse de réglages plus appropriés.

\section{Conclusion}

L'évolution des besoins industriels amène de plus en plus à l'élaboration de systèmes électrotechniques complexes, dont on attend de grandes performances dynamiques. Les conditions de fonctionnement auxquelles sont soumises les machines électriques dans ces applications s'éloignent parfois fortement des conditions nominales ; les modèles analytiques classiques sont alors 
a)
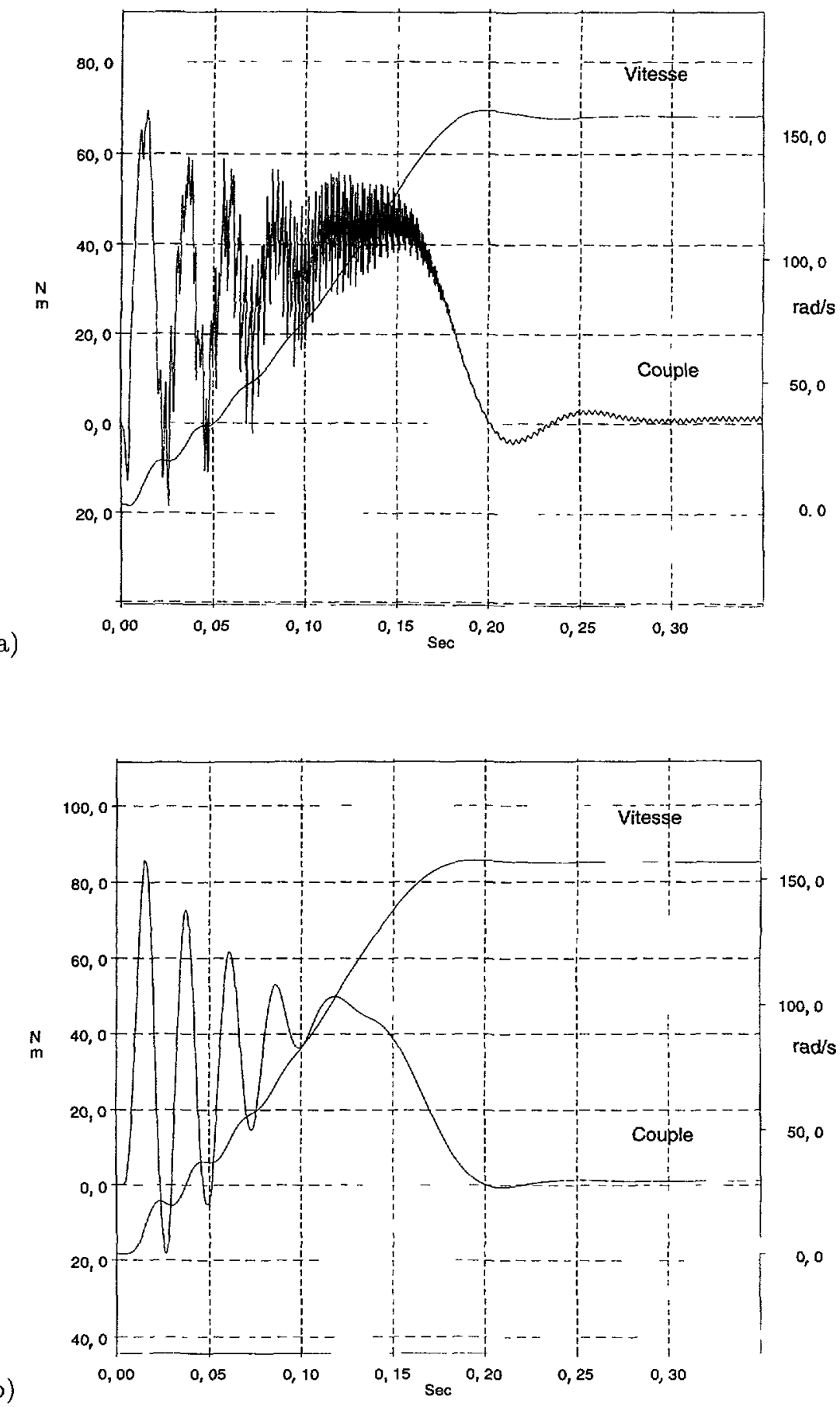

Fig. 22. - Couple et vitesse lors d'un démarrage à vide sous tension nominale. a) Simulation par la méthode des réseaux de perméances. b) Simulation par la méthode analytique.

[Torque and speed for starting at no-load under nominal voltage. a) Permeance network method results. b) Analytical method results.] 

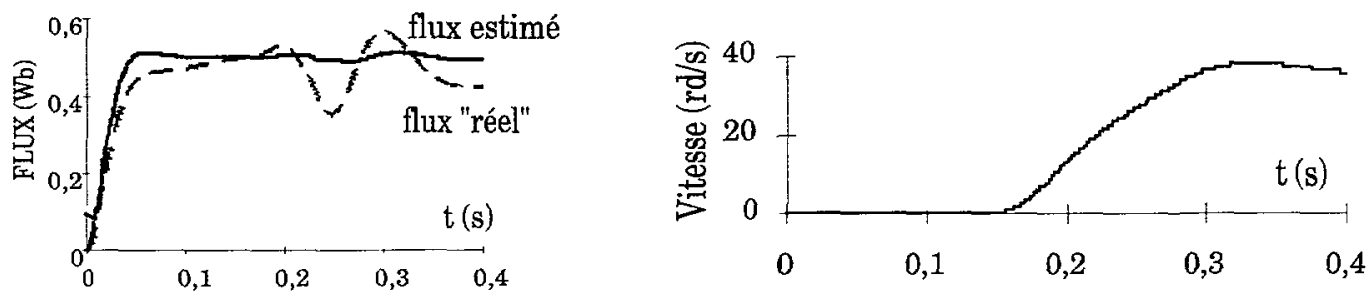

Fig. 23. - Flux estimé, flux "réel" et vitesse de rotation lors d'une réponse indicielle en vitesse.

[Estimated and "real" fluxes and speed during speed step.]

mis en défaut, et il est souhaitable d'adopter une modélisation plus fine de l'actionneur. Cependant, afin de pouvoir prendre en considération l'environnement électronique et mécanique du moteur, le modèle en question doit respecter un bon compromis précision/temps de calcul.

Pour répondre à ce cahier des charges, nous proposons une méthode de modélisation basée sur la description des systèmes électromagnétiques par réseau de perméances et la représentation bond-graphs. Cette méthode est explicitée puis testée dans le cas d'une machine asynchrone, branchée sur le réseau dans un premier temps, commandée vectoriellement dans un deuxième temps. Les résultats obtenus prouvent l'intérêt de la méthode : pour la modélisation de systèmes complexes faisant intervenir des ensembles commande, convertisseur, machine, charge, un maillon délicat reste la machine pour laquelle le modèle habituellement retenu est celui de Park. On connaît les limites de ce modèle, particulièrement pour les machines non conventionnelles. La méthode des réseaux de perméances permet de reculer ces limites en considérant la saturation et la géométrie de la machine. Associée à la méthode bond-graph, son champ d'applications peut encore s'étendre : en particulier on peut citer la possibilité de prise en compte des phénomènes thermiques facilitée par les propriétés de couplage des bond-graphs.

\section{Annexe A}

\section{Compléments sur la méthode bond-graph}

À chaque élément on peut associer une relation entre le flux et l'effort selon le formalisme suivant :

élément $R$ :

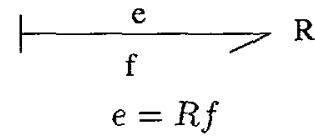

ou

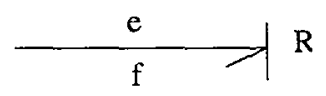

élément $C$ :

$$
f=e / R
$$

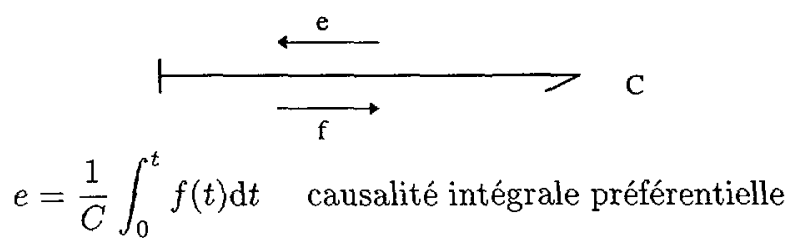


élément $I$ :

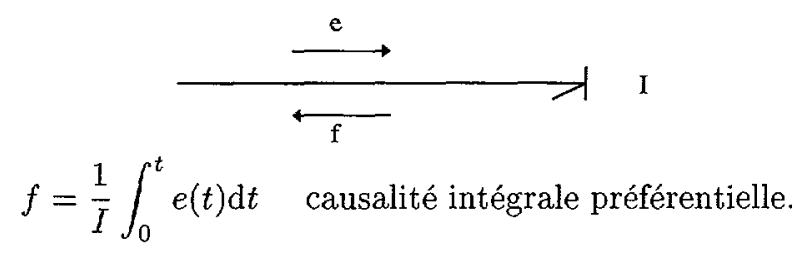

$f=\frac{1}{I} \int_{0}^{t} e(t) \mathrm{d} t \quad$ causalité intégrale préférentielle.

Les liaisons entre différents éléments sont du type "parallèle" ou "série" .

liaison série

jonction 1
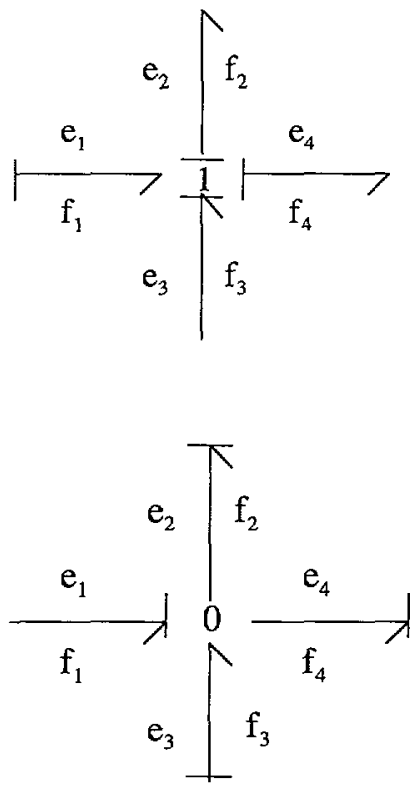

liaison parallèle jonction 0 les flux sont conservés et un seul élément peut imposer son flux

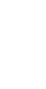




\section{Annexe B}

Valeur des paramètres de la machine asynchrone

$\begin{array}{lcc} & \text { essais expérimentaux } & \begin{array}{c}\text { méthode des } \\ \text { éléments finis }\end{array} \\ L_{\mathrm{cs}} \cdot \text { inductance cyclique statorique } & 205 \mathrm{mH} & 198 \mathrm{mH} \\ L_{\mathrm{cr}} \cdot \text { inductance cyclique rotorique } & 207,7 \mathrm{mH} & 198,2 \mathrm{mH} \\ M_{\mathrm{sr}} \cdot \text { inductance mutuelle cyclique stator/rotor } & 197,2 \mathrm{mH} & 190 \mathrm{mH} \\ r_{\mathrm{s}} \cdot \text { résistance d'une phase statorique } & 1,845 \Omega & \\ r_{\mathrm{r}} \cdot \text { résistance d'une phase rotorique } & 1,6 \Omega & \\ T_{\mathrm{s}}=\frac{L_{\mathrm{cs}}}{r_{\mathrm{s}}} \text { constante de temps statorique } & 111 \mathrm{~ms} & \\ T_{\mathrm{r}}=\frac{L_{\mathrm{cr}}}{r_{\mathrm{r}}} \text { constante de temps rotorique } & 130 \mathrm{~ms} & 8 \% \\ \sigma=1-\frac{M_{\mathrm{sr}}^{2}}{L_{\mathrm{cr}} L_{\mathrm{cs}}} \text { coefficient de dispersion } & 8,7 \% & \end{array}$

\section{Bibliographie}

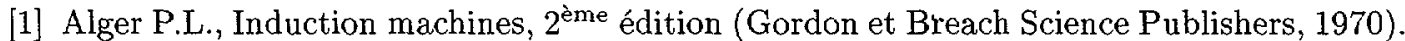

[2] Say M.G., Alternating current machine (Édition Pitman, 1976).

[3] Silvester P. and Chari M.V.K., Finite element solution of saturable magnetic field problems, IEEE Trans. 89 (1970) 1642-1651.

[4] Razek A., Coulomb J.L., Feliachi M. and Sabonnadière J.C., Conception of an air-gap element for dynamic analysis of the electromagnetic field in electric machines, IEEE Trans. Mag. 18 (1982) 655-659.

[5] Davat B., Lajoie-Mazenc M., Hector J. et Faucher J., Procédé d'analyse des systèmes électromagnétiques avec courants de Foucault : programme, DIFIMEDI, $R G E$ (Octobre 1982) 658-661.

[6] Ostovic V., Dynamics of saturated electric machines (Springer-Verlag, 1989).

[7] Karnopp D. and Rosenberg R., System Dynamics: a unified approach (John Wiley and Sons, 1975).

[8] Borne P., Dauphin-Tanguy G., Richard J.P., Rotella F. et Zambettakis I., Modélisation et identification des processus, Tome 2 (Éditions Technip, 1992).

[9] Delforge C., Modélisation d'un actionneur asynchrone et de sa commande vectorielle par réseaux de perméances, Thèse de l'UST (Lille, 20 Janvier 1995).

[10] Delforge C. and Lemaire-Semail B., Induction machine modeling using finite element and permeance network methods, IEEE Trans. Mag. 31 (May 1995) 2092-2095.

[11] Marrocco A., Analyse numérique des problèmes en électrotechnique, Ann. Sc. Math. Québec 1 (1977) 271-296.

[12] Jufer M., Électromécanique, Traité d'électricité, d'électronique et d'électrotechnique (Dunod, 1979).

[13] Cassat A., Étude numérique des caractéristiques statiques et du comportement dynamique des moteurs pas à pas polyphasés, Thèse de Doctorat (École Polytechnique Fédérale de Lausanne, 1977). 
[14] Nakhle M., Neptunix, an efficient tool for large size systems simulation, 2nd International Conf. on system simulation in building (Liège, Belgique, December 1986).

[15] Lemaire-Semail B., Brochet P. and Rombaut C., Asynchronous machine electromagnetic noise analysis using finite element method for determination of torque harmonics, ICEM 3 (Manchester, Septembre 1992) pp. 1005-1009.

[16] Ojo J.O., Consoli A. and Lipo T., An improved model of saturated induction machine, IEEE Trans. Industry Appl. 26 (March 1990).

[17] Leohnard W., Control of electrical drives (Springer Verlag, 1985).

[18] Hecquet M. and Brochet P., Modeling of a claw-pole alternator using permeance network coupled with electric cricuits, IEEE Trans. Mag. 31 (May 1995) 2131-2134.

[19] Carpenter C.J., Magnetic equivalent circuits, Proc. I.E.E. 115 (October 1968) 1503-1511.

[20] Laithwaite E.R., Magnetic equivalent circuits for electric machines, Proc. I.E.E. 14 (November 1967) 1805-1809.

[21] Slemon G.R., An equivalent circuit approach to analysis of synchronous machines with saliency and saturation, I.E.E.E. Trans. on Energy Conversion 5 (September 1990) 538545.

[22] Anuszczyk J., Rotational magnetization in magnetic circuits of electrical machines, Proc. ISEF'89, James and James Science Publishers (London, 1990) pp. 147-150.

[23] Xiao Y., Slemon G.R. and Iravani M.R., Implementation of an equivalent circuit approach to the analysis of synchronous machines, I.E.E.E. Trans. on Energy Conversion 9 (Dec. 1994) $717-723$. 